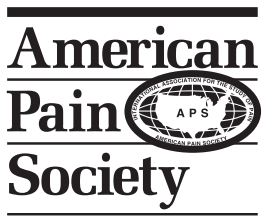

\title{
White Matter Involvement in Chronic Musculoskeletal Pain
}

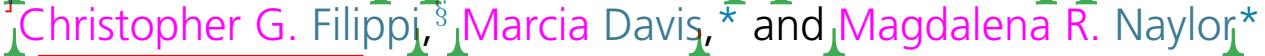

* University of Vermont,MindBody Medicine Clinic and Fletcher Allen Healthcare Clinical Neuroscience Research Unit, Burlington, Vermont.

$\dagger$ University of Vermont,Department of Radiology and MRI Center for Biomedical Imaging,|Burlington, Vermont. ${ }^{\ddagger}$ Philips Healthcare, Best, the Netherlands.

$\S$ Columbia University, Department of Radiology, New York, New York.

\begin{abstract}
There is emerging evidence that chronic musculoskeletal pain is associated with anatomic and functional abnormalities in gray matter. However, little research has investigated the relationship between chronic musculoskeletal pain and white matter. In this study, we used whole-brain tractbased spatial statistics and region-of-interest analyses of diffusion tensor imaging data to demonstrate that patients with chronic musculoskeletal pain exhibit several abnormal white matter integrity compared with healthy controls. Chronic musculoskeletal pain was associated with lower fractional anisotropy in the splenium of the corpus callosum and the left cingulum adjacent to the hippocampus. Patients also had higher radial diffusivity in the splenium, right anterior and posterior limbs of the internal capsule, external capsule, superior longitudinal fasciculus, and cerebral peduncle. Patterns of axial diffusivity (AD) varied: Patients exhibited lower AD in the left cingulum adjacent to the hippocampus and higher $A D$ in the anterior limbs of the internal capsule and in the right cerebral peduncle. Several correlations between diffusion metrics and clinical variables were also significant at a $P<.01$ level: Fractional anisotropy in the left uncinate fasciculus correlated positively with total pain experience and typical levels of pain severity. AD in the left anterior limb of the internal capsule and left uncinate fasciculus were correlated with total pain experience and typical pain level. Positive correlations were also found between $A D$ in the right uncinate and both total pain experience and pain catastrophizing. These results demonstrate that white matter abnormalities play a role in chronic musculoskeletal pain: either as a cause, predisposing factor, consequence, or compensatory adaptation.

Perspective: Patients with chronic musculoskeletal pain exhibit altered metrics of diffusion in the brain's white matter compared with healthy volunteers, and some of these differences are directly related to symptom severity.

(C) 2014 by the American Pain Society

Key words: DTI, white matter, chronic pain, neuroimaging.
\end{abstract}

usculoskeletal pain syndromes such as chronic back pain and osteoarthritis are some of the most common forms of chronic pain. ${ }^{22}$ There is extensive evidence that abnormalities exist in the brains

Received December 10, 2013; Revised July 7, 2014; Accepted August 3, 2014.

Funded by National Institute of Arthritis and Musculoskeletal and Skin Q2] Diseases (NIAMS) grant R01-AR059674 (to M.R.N.), the UVM MRI Center for Biomedical Imaging, and U.S. Department of Energy grant [Q3] SC0001753.

The authors report no conflicts of interest.

Address reprint requests to Magdalena R. Naylor, MD, PhD, Clinical Neuroscience Research Unit, Department of Psychiatry, University of Vermont, 1 South Prospect Street, UHC/Arnold 6, Burlington, VT 05401. E-mail: Magdalena.Naylor@uvm.edu

$1526-5900 / \$ 36.00$

(C) 2014 by the American Pain Society

http://dx.doi.org/10.1016/j.jpain.2014.08.002 of chronic musculoskeletal pain patients in gray matter (GM) volume/thickness, ${ }^{13,15,27,40,44,49,57,61 \quad \text { GM }}$ density, $^{7,9,60,62,64,65}$ and both acute pain-related ${ }^{16,19}$ and resting state $8,28,38,43$ functional activity. Although some work has been published regarding the relationship between chronic pain disorders and neural white matter (WM), ${ }^{12,18,21,25,26,44,45,50,72,73}$ only 1 study $^{50}$ has used contemporary analysis approaches to compare measures of anisotropy and diffusion specifically between chronic musculoskeletal pain patients with temporomandibular disorder (TMD) and healthy volunteers. Moayedi and colleagues showed that patients have highly significant clusters of lower fractional anisotropy (FA) and higher radial diffusivity (RD) in the trigeminal nerves, right internal capsule, and right external/extreme capsule and diffusely throughout 


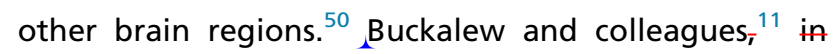
another article, did not compare patients with healthy volunteers but did show that patients with disabling chronic back pain have lower FA in the splenium of the corpus callosum compared with patients with nondisabling chronic back pain, which correlated with pain duration. Overall, diffusion tensor imaging (DTI) findings have so far been inconsistent across studies of other types of chronic pain (eg, fibromyalgia, ${ }^{44,72}$ chronic complex regional pain syndrome, ${ }^{26}$ migraine ${ }^{73}$ irritable bowel syndrome, ${ }^{18,21}$ and chronic pancreatitis ${ }^{25}$ ), and WM integrity in chronic musculoskeletal pain remains a relatively poorly explored research topic. In addition, the generalizability of findings in TMD to other types of musculoskeletal pain is currently unknown.

The objectives of this study are to determine whether there are differences in pain-related WM pathways in the brains of a broad sample of chronic musculoskeletal pain patients (including diagnoses of osteoarthritis and back, limb, and abdominal muscle pain) compared with healthy volunteers. We hypothesized that chronic musculoskeletal pain patients would exhibit lower FA within specific fiber tracts shown to be involved in TMD compared with healthy controls and in other musculoskeletal pain disorders using different experimental approaches: the splenium of the corpus callosum, ${ }^{11,45,50}$ the anterior and posterior limbs of the internal capsule, ${ }^{45,50}$ the cingulum bundle, ${ }^{50}$ the temporal lobe branch of the cingulum, ${ }^{12}$ the external capsule adjacent to the insular cortex, ${ }^{45}$ and the superior longitudinal fasciculus. ${ }^{12,45}$ Additional exploratory analyses were performed on the uncinate fasciculus and the cerebral peduncles because of their involvement in emotional processing and descending motor signaling and pain inhibition, respectively.

Identifying WM abnormalities in a broad sample of chronic musculoskeletal pain patients that overlap with reported differences in TMD and/or nonmusculoskeletal pain disorders may provide insight into which fiber pathways play a role specifically in chronic musculoskeletal pain. Additionally, because abnormalities in WM pathways reflect changes in tract integrity between brain regions known to process the somatosensory, affective, and cognitive components of pain perception, we predicted that FA within these tracts would correlate with specific representative clinical measures of pain symptom severity.

\section{Methods}

\section{Participant Recruitment and Initial Evaluation}

All research protocols were reviewed and approved by the University's institutional review board. Forty-six patients between the ages of 18 and 65 years with primary chronic musculoskeletal pain diagnoses and 33 age-matched healthy volunteers were analyzed for this cross-sectional study. These participants were pulled from a larger pool of participants recruited for a series of longitudinal experiments.
Participants were evaluated in person for study eligibility; they provided informed consent and underwent a comprehensive clinical assessment of chronic pain symptoms, demographics, and cognitive eligibility based on the Wide Range Achievement Test ${ }^{34}$ and the Mini-Mental State Examination. ${ }^{24}$ Eligibility requirements included at least 1 year of self-reported chronic musculoskeletal pain symptoms scored at 4 or higher on a scale from 0 to 10 (where 0 represented no pain and 10 the worst pain). Exclusion criteria included inability to participate in magnetic resonance imaging (eg, claustrophobia, ferrous metal in the body), opiate medication use, previous history of traumatic brain injury, uncontrolled/unmedicated diabetes or hypertension, and comorbid diagnoses of psychiatric disorders (eg, current major depression, bipolar disorder, and schizophrenia). Primary chronic musculoskeletal pain diagnoses included osteoarthritis and postinjury back, neck, shoulder, knee/leg, and abdominal muscle pain. Several patients had a medical history containing more than 1 chronic musculoskeletal pain diagnosis.

\section{Clinical Assessment}

A preliminary phone screening, an initial in-person clinical evaluation, and a series of formal, selfadministered, previously validated questionnaires were used to assess measures of pain, function, disability, and mental health for all patients. The initial clinical evaluation included questions regarding demographics, diagnoses, current and typical levels of pain, medical history, and treatment/medication management. Pain, function, and disability were assessed using the short form of the McGill Pain Questionnaire (MPQ) ${ }^{46,48}$ and the pain symptoms subscale of the Treatment Outcomes in Pain Survey (TOPS). ${ }^{58,59}$ Depression was assessed using the Beck Depression Inventory. ${ }^{10}$ Additional measures of coping skills use, ability to control pain, ability to decrease pain, and degree of pain catastrophizing were collected using the Pain Catastrophizing Scale ${ }^{71}$ and the Chronic Pain Self-Efficacy Scale. ${ }^{3}$ Healthy volunteer participants were asked to complete a demographics questionnaire and the Beck Depression Inventory. Clinical data were doubleentered and reconciled, scored according to the instructions for each questionnaire, and stored and managed using REDCap electronic data capture tools. ${ }^{29}$

\section{DTI Acquisition}

DTI data were acquired using a Philips Achieva $3 T$ magnet (Philips Healthcare, Best, the Netherlands) with an 8-channel head coil, utilizing the following parameters: axial 2-dimensional spin echo echo-planar imaging sequence with 46 diffusion directions, 59 slices, 2-mm slice thickness, 10,000 -ms repetition time, $68 \mathrm{~ms}$ echo time, $2 \times 2-\mathrm{mm}$ in-plane resolution, $b=1,000 \mathrm{~s} / \mathrm{mm}^{2}$, flip angle of $90^{\circ}$, and an echo planar imaging factor of 63. An axial T2-weighted gradient spin echo sequence was also acquired for radiological review in order to rule out neurologically significant abnormalities and pathology. 


\section{DTI Analysis}

DTI data were processed using the Oxford Centre for Functional MRI of the Brain (FMRIB) Software Library's ${ }^{35,70,79}$ Diffusion Toolbox. Data underwent eddy current correction, brain extraction, ${ }^{68}$ and fitting with a diffusion tensor model at each voxel in order to_falculate FA, RD, and axial diffusivity (AD) maps. All raw images and processed FA maps were visually inspected for artifacts, excessive motion, and anatomic abnormalities that might interfere with registration such as lesions or enlarged cerebral ventricles due to cortical atrophy. ${ }^{37}$ FA maps were nonlinearly registered ${ }^{5,6,36}$ into $1 \times 1 \times 1$-mm standard space (FMRIB58_FA) and affinealigned into MNI152 space. FA data were projected onto an alignment-invariant mean FA skeleton representing the centers of each tract in the brain before voxelwise cross-subject statistics were applied using tract-based spatial statistics (TBSS). ${ }^{69}$ Skeletonization was carried out using an FA threshold of .2 in order to exclude GM and cerebrospinal fluid.

Between-group voxelwise comparisons were performed Q5] using randomize, ${ }^{4,14,31}$ a nonparametric permutation test that utilizes threshold-free cluster enhancement to correct for multiple comparisons. Ten thousand permutations were performed per t-test. RD and AD images were processed using the same nonlinear registration steps and mean WM skeleton derived for FA. Participant ages were de-meaned and input into the model as covariates. To improve visualization of thresholded TBSS results, clusters were thickened into local WM tracts.

DTI data from specific regions of interest were also analyzed individually. Binary WM masks were created from the Johns Hopkins University ICBM-DTI-81 atlas of WM labels, ${ }^{32,51,52}$ which was produced from average DTI maps from 81 participants with a mean age of 39 years. Specific tracts selected for analysis based on the existing literature included the splenium of the corpus callosum, the anterior and posterior limbs of the internal capsule, the external capsule adjacent to the insular cortex, the cingulum bundle, the temporal lobe branch of the cingulum bundle adjacent to the hippocampus, the superior longitudinal fasciculus, and the uncinate fasciculus. Additionally, post hoc regionof-interest analyses were performed on the cerebral peduncles after clusters of significantly higher RD were observed there in patients using TBSS. Mean FA, RD, and $A D$ values were extracted from whole brain and from specific tracts by applying the binary masks to each volume (specific participant) of the skeletonized TBSS output. Skeletonized output was used rather than nonskeletonized in order to improve consistency across subjects and to ensure that only voxels from the WM were compared. Correlations between DTI data and clinical measures were performed using predetermined clinical measures thought to best represent pain severity and negative impact on life: pain duration, MPQ typical level of pain, TOPS total pain experience, and the Pain Catastrophizing Scale.

All region-of-interest DTI analyses and clinical comparisons were performed using SPSS Statistics for Windows, version 20 (IBM Corporation, Armonk, NY). Graphs were created using Microsoft Excel and GraphPad Prism for Windows, version 6.00 (GraphPad Software, La Jolla, CA).

\section{Results}

\section{Clinical Assessment of Chronic Musculoskeletal Pain Severity}

Forty-six patients with chronic musculoskeletal pain (12 male, 34 female) were compared to 33 agematched healthy control volunteers ( 14 male, 19 female). There was no significant difference in age $(P=.45)$ between patients (mean $=45.8$, standard deviation $[S D]=12.9$ ) and healthy controls (mean $=44.5$, $S D=13.3$ ). Patients reported a mean chronic pain duration of 9.85 years $(S D=8.12)$ and a mean typical pain level of 5.5 (SD = 2.12) on a Likert-type scale ranging from $0=$ no pain to $10=$ the worst pain. Patients also reported a mean current pain level of $5.2(S D=2.4)$ at the time of questionnaire administration. Total pain experience and perceived family disability (a measure of pain interference) composite scores from TOPS were reported as $52.17(S D=16.39)$ and $49.97(S D=18.84)$, respectively, on a scale from 0 to 100 , whereas the mean Pain Catastrophizing Scale score across the patient group was 19.47 (SD =11.47) on a scale from 0 to 50 . The mean score on the Beck Depression Inventory was 14.28 ( $S D=9.88$ ) on a scale from 0 to 63 , which is considered to be mild depression. ${ }^{10,64}$ These measures fall within ranges that would be expected in a sample of chronic pain patients. ${ }^{53,54,64}$ Healthy volunteers did not report current chronic pain and were not depressed (BDI mean $=3.4, \mathrm{SD}=4.50$ ).

\section{Whole-Brain TBSS Analyses}

TBSS analysis of skeletonized FA and AD data yielded no statistically significant differences between chronic musculoskeletal pain patients and healthy controls at a significance level of $P<.05$ using threshold-free cluster enhancement to correct for multiple comparisons. A number of regions did exhibit nonsignificantly lower FA $(P<.10)$ in patients than controls, however, including several of the a priori regions of interest discussed below.

TBSS analysis of skeletonized RD data yielded a number of statistically significant differences between groups. Clusters of higher RD in patients were present in the body of corpus callosum, right superior longitudinal fasciculus, anterior and posterior limbs of the internal capsule, and the external capsule (Fig 1) as well as in the splenium of the corpus callosum, the right cingulum adjacent to the hippocampus, and the right cerebral peduncle (Fig 2). Each of these clusters was significant at a threshold of $P=.05$, fully corrected for multiple comparisons using threshold-free cluster enhancement. No clusters were identified that exhibited lower RD in patients than in controls. Specific region-of-interest analyses were also used to investigate the relationship between RD within specific tracts in chronic musculoskeletal pain and clinical measures. $F A$ and $A D$ values were 
4 The Journal of Pain comp: web and color in print

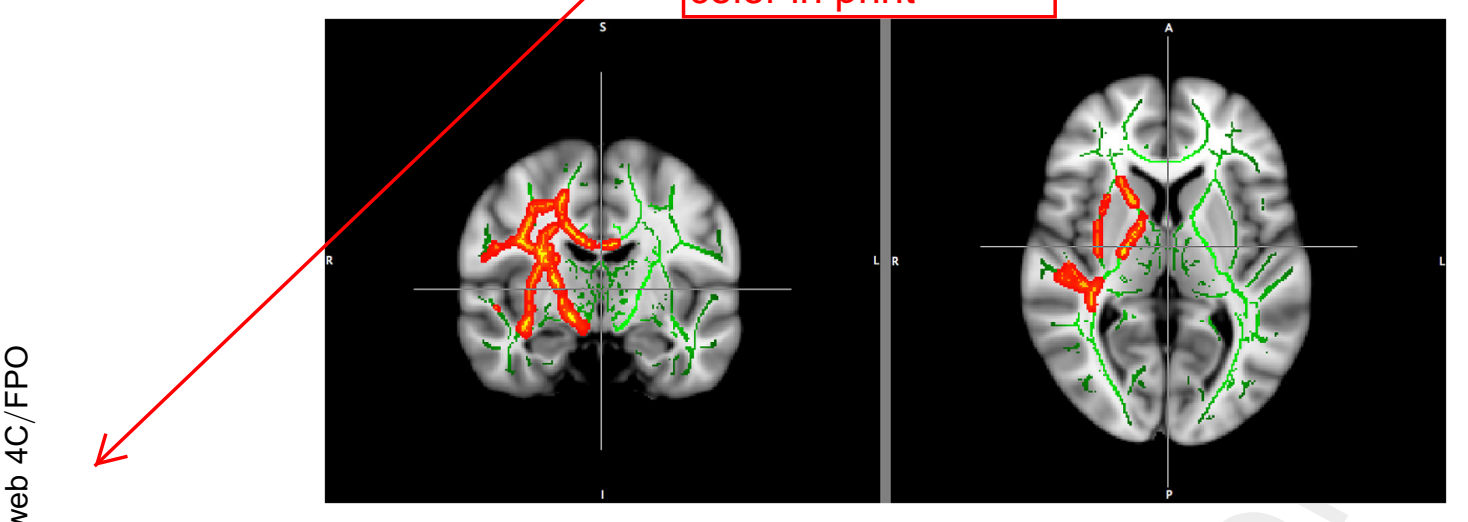

Figure 1. Coronal (left) and axial view (right) of TBSS analysis of RD differences between chronic musculoskeletal pain patients and healthy control participants, at the level of the thalamus. A large cluster of higher RD contained fibers of the corpus callosum, right superior longitudinal fasciculus, right anterior and posterior limbs of the internal capsule, and right external capsule adjacent to the insular cortex. Clusters are significant at a level of $P<.05$ after correcting for multiple comparisons using threshold-free cluster enhancement.

also extracted and analyzed in order to provide further insight into the local microstructural properties of painrelated fiber pathways.

\section{Region-of-Interest Analyses}

Analysis of mean $F A, R D$, and $A D$ values extracted from binary masks of specific WM tracts revealed a number of differences between chronic musculoskeletal pain patients and healthy volunteers. Because there were significant effects of age and gender on many of the diffusion measures, both measures were entered into the general linear model as covariates. All statistics reported below were generated by analyzing the effect of group after controlling for both age and gender.

Several tracts were identified that exhibited lower FA in chronic musculoskeletal pain patients compared with healthy controls, whereas no regions were identified that exhibited higher FA in patients (Fig 3). Specific tracts that exhibited lower FA in patients included the splenium of the corpus callosum ( $F=4.647, P=.034)$ and the left temporal lobe branch of the cingulum bundle adjacent to the hippocampus $(F=4.450, P=.038)$.

Consistent with the TBSS results reported above, several tracts exhibited higher RD in patients compared with healthy volunteers, and no tracts exhibited lower RD in patients (Fig 4). Higher RD was observed in patients in the splenium of the corpus callosum $(F=6.499$, $P=.013)$ and in the right anterior $(F=4.018, P=.049)$ and posterior $(F=6.346, P=.014)$ limbs of the internal capsule, the external capsule adjacent to the insular cortex $(F=7.400, P=.008)$, the superior longitudinal fasciculus $(F=7.946, P=.006)$, and the cerebral peduncle $(F=5.652, P=.020)$. No difference was observed in the region-of-interest analysis of the body of the corpus callosum, possibly because the cluster observed there using TBSS was relatively small in relation to the volume of the entire tract.

Finally (Fig 5), the left temporal lobe branch of the cingulum exhibited lower $A D$ in patients than controls $(\mathrm{F}=4.404, P=.039)$, and 3 specific tracts demonstrated higher $A D$ in chronic musculoskeletal pain patients: the right cerebral peduncle $(F=4.423, P=.039)$ and both the right $(F=17.430, P<.001)$ and left $(F=5.528$, $P=.021)$ anterior limbs of the internal capsule.

\section{Clinical Correlations}

Exploratory correlational analyses were performed comparing representative measures of pain severity

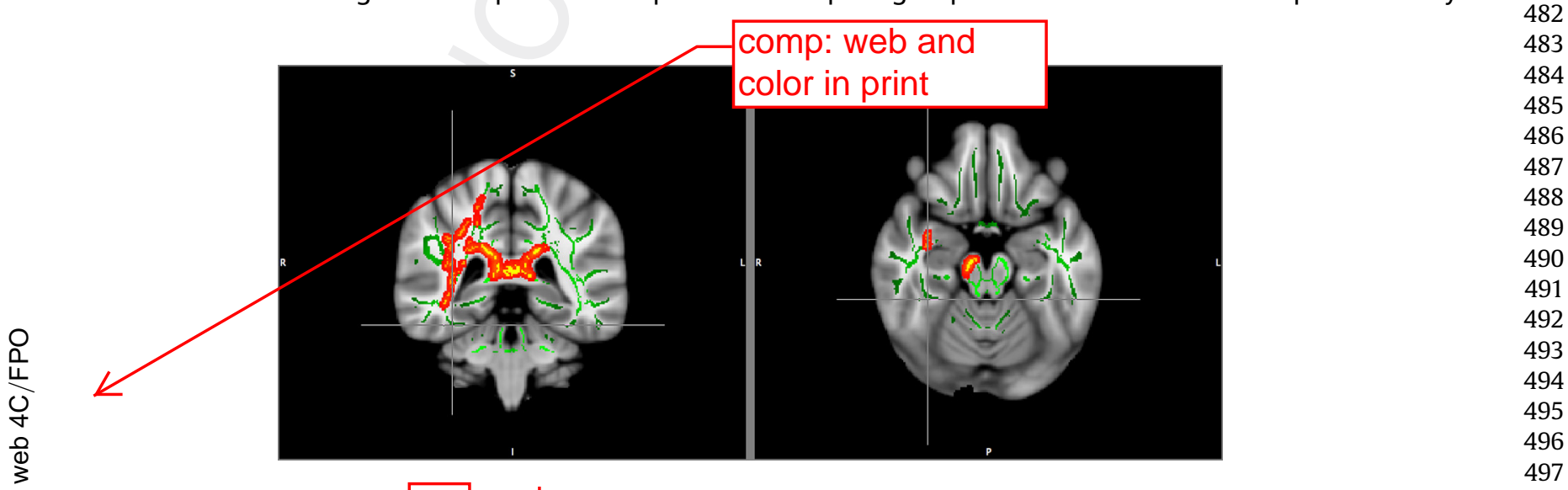

Figure 2. Coronal (left) and axial|view (right) of TBSS analysis of RD differences between chronic musculoskeletal pain patients and healthy control participants, at the level of the thalamus. Cluster of higher RD contained fibers of the splenium of the corpus callosum, right superior longitudinal fasciculus, right cingulum bundle adjacent to the hippocampus, and right cerebral peduncle. Clusters are significant at a level of $P<.05$ after correcting for multiple comparisons using threshold-free cluster enhancement. 


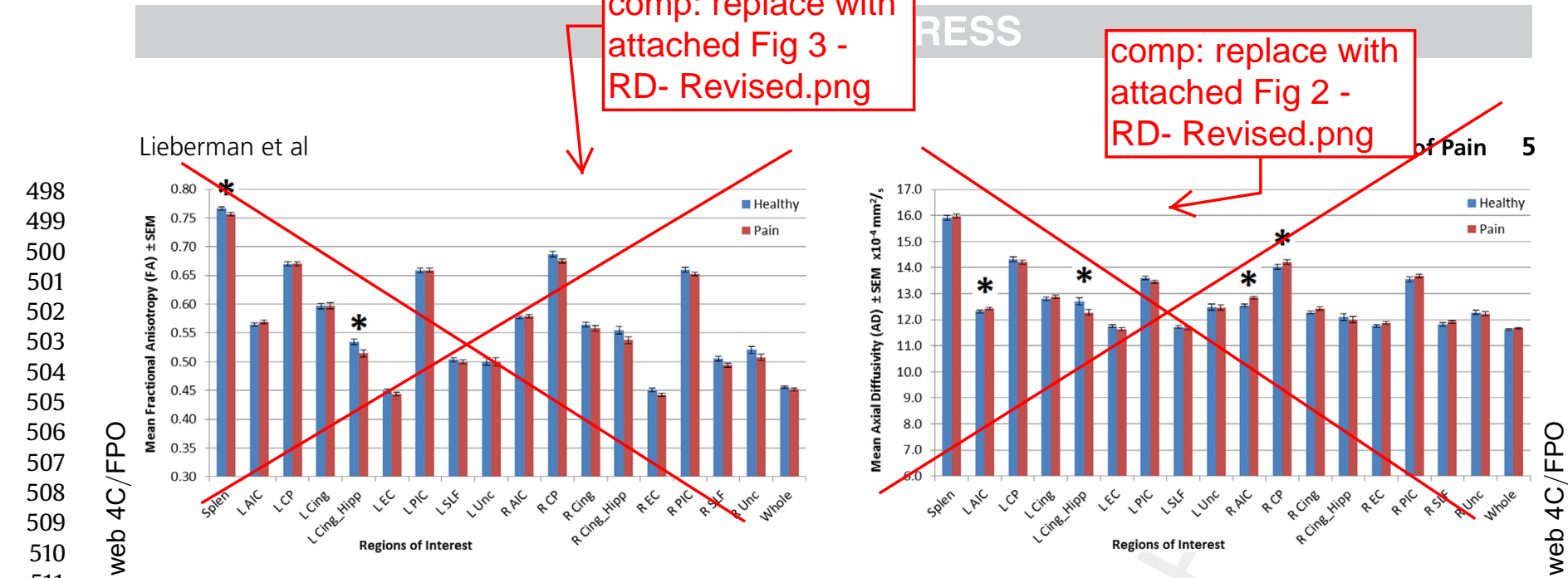

Figure 3. Comparison of mean FA values extracted from specific pain-related cognitive, limbic, and sensory tracts between chronic musculoskeletal pain patients and healthy volunteers. Contrasts designated with an asterisk were significant at a level of $P<.05$ after correcting for both age and gender. Error bars signify the SEMs. Abbreviations: SEM, standard error of the mean; Splen, splenium of the corpus callosum; L, left; $A I C$, anterior limb of the internal capsule; $C P$, cerebral peduncle; Cing, cingulum bundle; Cing_Hipp, temporal lobe branch of the cingulum bundle adjacent to the hippocampus; EC, external capsule; PIC, posterior limb of the internal capsule; SLF, superior longitudinal fasciculus; Unc, uncinate fasciculus; $\mathrm{R}$, right; Whole, whole brain.

and negative impact on life with mean $F A, R D$, and $A D$ values extracted from specific regions of interest in the brains of chronic musculoskeletal pain patients. Because of the exploratory nature of this analysis and lack of correction for multiple comparisons, only correlations that were significant at a $P<.01$ level are reported.

Despite there being no significant between-group difference, significant positive correlations (Fig 6) were found between FA in the left uncinate fasciculus and the TOPS total pain experience composite score $(r=.399, P=.006)$ and self-reported typical pain levels $(r=.415, P=.004)$.

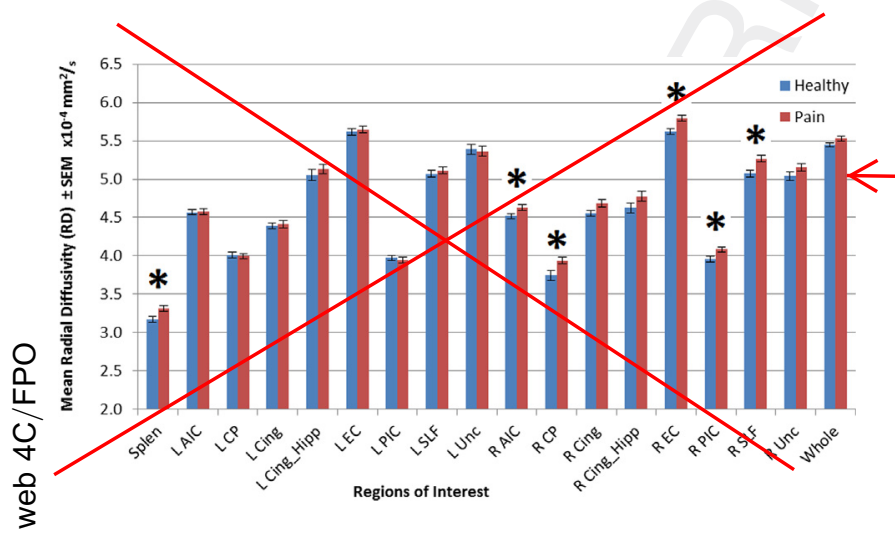

Figure 4. Comparison of mean RD values extracted from specific pain-related cognitive, limbic, and sensory tracts between chronic musculoskeletal pain patients and healthy volunteers. Contrasts designated with an asterisk were significant at a level of $P<.05$ after correcting for both age and gender. Error bars signify the SEMs. Abbreviations: SEM, standard error of the mean; Splen, splenium of the corpus callosum; L, left; $\mathrm{AIC}$, anterior limb of the internal capsule; $\mathrm{CP}$, cerebral peduncle; Cing, cingulum bundle; Cing_Hipp, temporal lobe branch of the cingulum bundle adjacent to the hippocampus; EC, external capsule; PIC, posterior limb of the internal capsule; SLF, superior longitudinal fasciculus; Unc, uncinate fasciculus; $\mathrm{R}$, right; Whole, whole brain.
Figure 5. Comparison of mean $A D$ values extracted from specific pain-related cognitive, limbic, and sensory tracts between chronic musculoskeletal pain patients and healthy volunteers. Contrasts designated with an asterisk were significant at a level of $P<.05$ after correcting for both age and gender. Error bars signify the SEMs. Abbreviations: SEM, standard error of the mean; Splen, splenium of the corpus callosum; L, left; $\mathrm{AIC}$, anterior limb of the internal capsule; $\mathrm{CP}$, cerebral peduncle; Cing, cingulum bundle; Cing_Hipp, temporal lobe branch of the cingulum bundle adjacent to the hippocampus; EC, external capsule; PIC, posterior limb of the internal capsule; SLF, superior longitudinal fasciculus; Unc, uncinate fasciculus; $R$, right; Whole, whole brain.

No correlations were observed between clinical measures and RD at a significance level of .01, though 6 significant positive correlations were observed between clinical measures and AD (Fig 7). TOPS total pain experience was correlated with $A D$ in the left uncinate fasciculus $(r=.393, P=.007)$ and the left anterior limb of the internal capsule $(r=.398, P=.006)$. AD in the left posterior limb of the internal capsule also demonstrated a correlation with total pain experience, though not at a significance level of $P<.01(r=.373$, $P=.011)$. MPQ typical pain demonstrated significant correlations with $A D$ in the same 2 regions:-left uncinate $(\mathrm{r}=.480, P=.001)_{\overline{\underline{\alpha}}}$ left anterior $\operatorname{limb}(f=.380, P=.009)_{\tau_{\bar{\alpha}}}$ as well as the right uncinate fasciculus $(r=.451, P=.002)$. Finally, $A D$ in the right uncinate also was correlated with pain catastrophizing $(r=.381, P=.010)$.

\section{Discussion} comp: replace with attached Fig 4

In this study, we demonstrate that patients with chronic musculoskeletal pain exhibit significant WM differences in $F A, R D$, and $A D$ compared with healthy volunteers and that the several differences in measures of diffusion are correlated with symptom severity. It has been known for some time that abnormal GM organization and altered patterns of functional activity are associated with chronic pain, and the results presented here show that WM plays an important role in chronic musculoskeletal pain, as well. The majority of the tracts that exhibited abnormal WM properties in this study convey information regarding specific components of pain perception-somatosensory, cognitive, and/or emotional-whereas others seem to be related to motor pathways and tracts involved in descending inhibition of pain.

Assessing not only FA but also RD and AD within specific tracts is important because these measures reflect 
6 The Journal of Pain

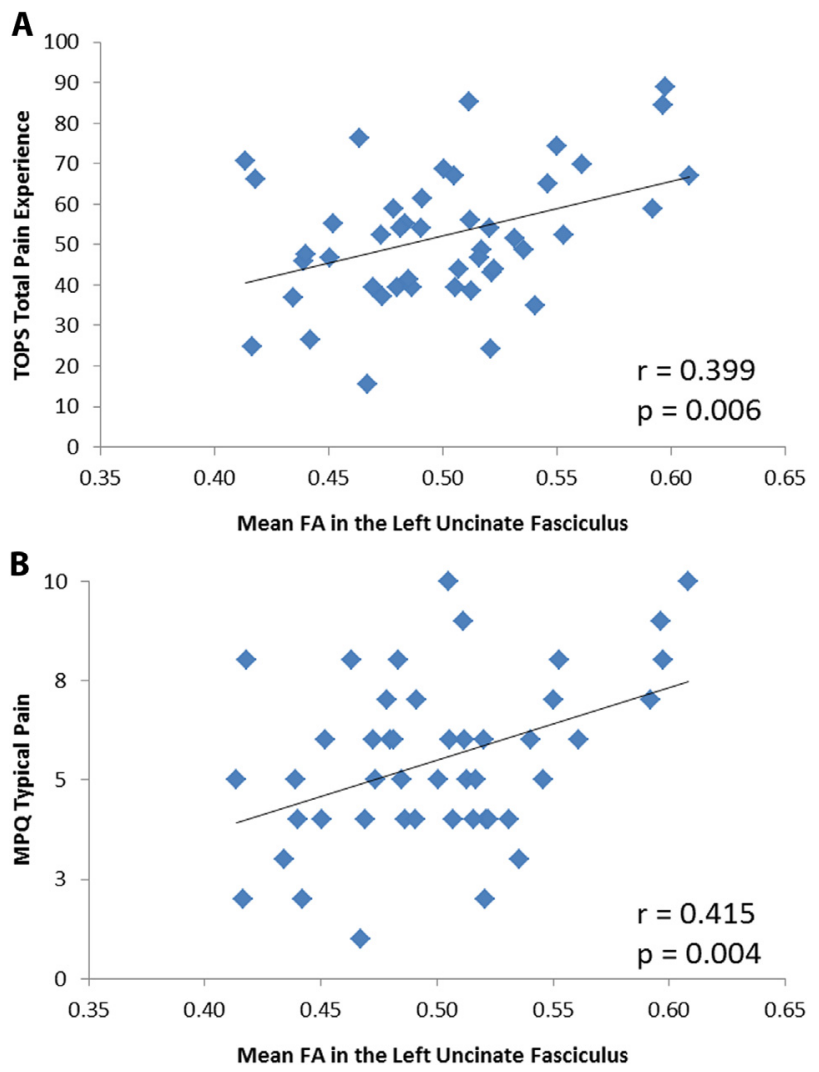

Figure 6. Exploratory correlational relationships between FA and clinical measures. FA in the left uncinate fasciculus positively correlated with both (A) TOPS total pain experience and (B) MPQ typical pain rating.

microstructural properties that represent different aspects of tract integrity. Specifically, WM neuropathology is frequently associated with decreased FA within specific tracts, ${ }^{2}$ but it is unclear whether lower FA is due to differences in fiber density, axon thickness, plasticity-related degree of myelination, or something else entirely. Because FA is a function of the amount of $A D$ in relation to the amount of RD, it is important to determine whether FA differences are driven by differences in $R D, A D$, or both. For example, the lower FA observed in the splenium of the corpus callosum in chronic musculoskeletal pain patients is associated with lower RD (and no significant difference in $A D$ ), whereas the lower FA observed in the left cingulum adjacent to the hippocampus is associated with higher $A D$ (and no difference in RD). It is equally important to note that the lack of significant difference observed in FA in the right anterior limb of the internal capsule may be due to the observation that both $R D$ and $A D$ are higher in this tract in patients than in healthy volunteers. It has been suggested that changes in RD likely reflect degree of myelination (as myelin restricts radial diffusion), whereas changes in $A D$ are less well understood and might reflect anything from axon thickness to fiber density. Although much additional work is needed in order to understand the mechanisms behind these differences and the nature of any causative relationships that may exist, it seems clear that pain-related abnormalities in
FA can be a consequence of changes in diffusion both axially and radially within specific tracts (Table 1). Especially considering that clinical correlations were observed with multiple measures of diffusion within various tracts, additional work is needed in order to understand the mechanisms by which $R D$ and $A D$ are involved in chronic musculoskeletal pain as well as the implications for diagnosis, prevention, and/or treatment of chronic musculoskeletal pain disorders.

The splenium of the corpus callosum contains fibers that connect regions of the parietal cortex that process somatosensory information and several temporal lobe structures ${ }^{17}$ in addition to visual information. Our finding that FA is lower (and RD is higher) in this region may reflect abnormal processing of somatosensory or nociceptive input from the periphery. This result is supported by previous research demonstrating lower splenium WM integrity in patients with migraine ${ }^{42}$ as well as patients with disabling chronic back pain compared with patients with nondisabling chronic back pain. ${ }^{11}$

The temporal lobe branch of the cingulum conveys fibers from the cingulate cortex, one of the principal processing centers of the cognitive dimensions of chronic pain, to the entorhinal cortex and then to the hippocampus, possibly playing a role in the emotional memories and learned behaviors associated with chronic pain. ${ }^{56,67,77,78}$ Neurons in the cingulate cortex have been shown to respond to both thermal and mechanical pain. ${ }^{33}$ Our finding of lower FA and $A D$ in this tract may reflect abnormal structural connectivity between pain-processing centers in the anterior cingulate cortex and temporal lobe memory/limbic regions, contributing to or resulting from chronic pain-related neuropathology.

The anterior limbs of the internal capsule contain fibers that project from the medial thalamus (which receives ascending nociceptive information from the periphery) to the prefrontal cortex, a tract likely involved in processing the cognitive and attention-related components of pain perception. Our finding that RD is higher in this region in patients with chronic musculoskeletal pain (interestingly in conjunction with higher $A D$ ) is consistent with findings in $T M D,{ }^{50}$ supporting conclusions by Mansour and colleagues that it can serve as a predictor of development of chronic pain after back injury. ${ }^{45}$

The external capsule provides some of the input to the insular cortex and conveys information pertaining to the emotional component of pain perception. ${ }^{18,63,75}$ The present demonstration of elevated RD in this region in chronic musculoskeletal pain is consistent with previous research ${ }^{45,50}$ and bolsters the argument that decreased tract integrity of insular inputs may play a role in pathologic perception of pain. Contradictory to these results, FA in the external capsule has been shown to be elevated in irritable bowel syndrome, a chronic visceral pain disorder, and correlated with bowel pain duration. ${ }^{18} \mathrm{It}$ is unclear whether these differences are anomalies due to different measurement techniques or unique properties of specific subtypes of chronic pain. 
Lieberman et al

The Journal of Pain

7
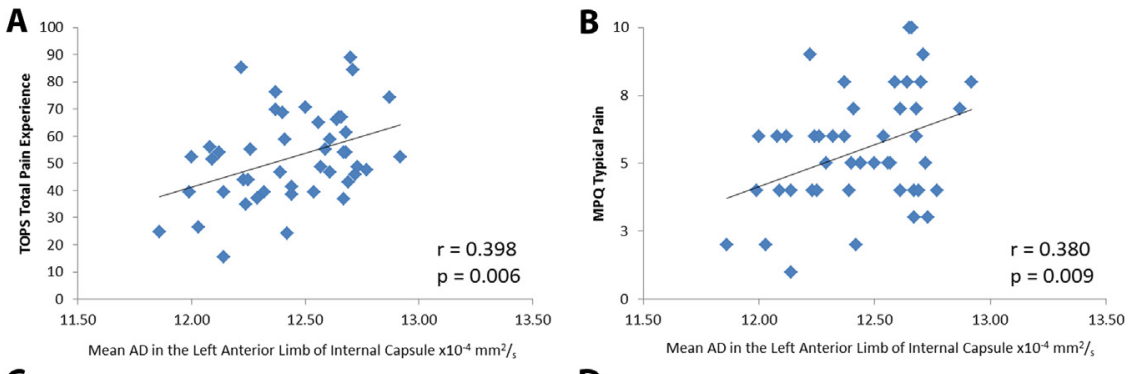

C
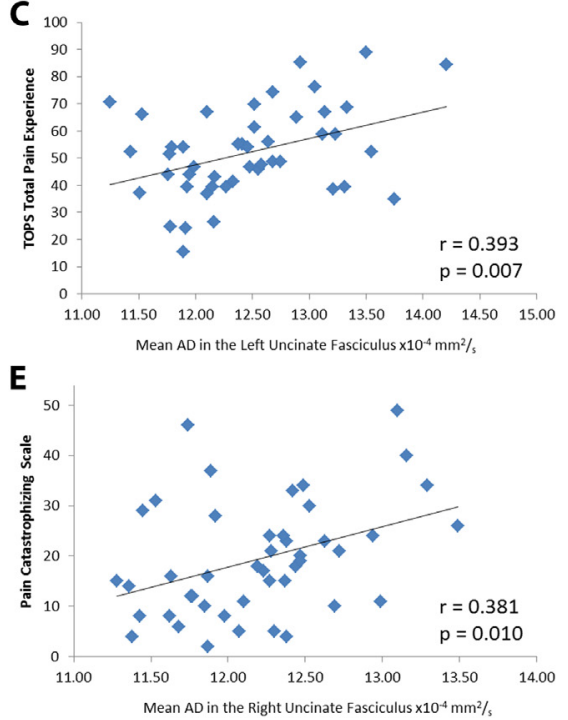

D 10

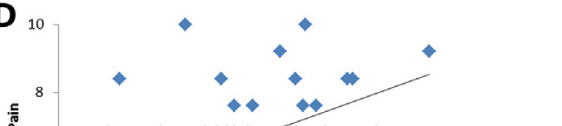

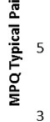

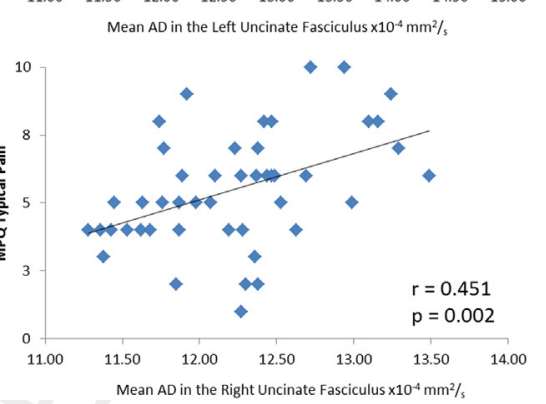

F $_{10}$

Figure 7. Exploratory correlational relationships between $A D$ and clinical measures. $A D$ in the left uncinate anterior limb of the internal capsule positively correlated with both (A) TOPS total pain experience and (B) MPQ typical pain rating, as did AD within the left uncinate fasciculus (C, D). AD within the right uncinate fasciculus also positively correlated with both (E) Pain Catastrophizing Scale score and (F) MPQ typical pain rating.

The exact functions of the uncinate fasciculus are not yet fully known, but it has been shown to connect temporal lobe structures such as the amygdala and hippocampus with cognitive processing centers in the prefrontal cortex..$^{30}$ The uncinate demonstrates abnormal diffusion properties in a number of psychological

\section{Table 1. Patterns of FA, RD, and AD: Chronic Musculoskeletal Pain Patients Versus Healthy Controls}

\begin{tabular}{lccc}
\hline \multicolumn{1}{c}{ REGION OF INTEREST } & $F A$ & $R D$ & $A D$ \\
\hline Splenium of the corpus callosum & $\downarrow$ & $\uparrow$ & \\
Left cingulum, temporal & $\downarrow$ & & $\downarrow$ \\
Left anterior limb, internal capsule & & & $\uparrow$ \\
Right anterior limb, internal capsule & & $\uparrow$ & $\uparrow$ \\
Right posterior limb, internal capsule & $\uparrow$ & \\
Right external capsule & & $\uparrow$ & \\
Right superior longitudinal fasciculus & & $\uparrow$ & \\
Right cerebral peduncle & $\uparrow$ & $\uparrow$ \\
\hline
\end{tabular}

NOTE. The table demonstrates the relationship between FA, RD, and AD within specific cognitive, limbic, and sensory pathways of the pain matrix. Upwardpointing arrows indicate metrics that are higher in chronic musculoskeletal pain patients than healthy control participants, and downward-pointing arrows indicate the reverse. In most cases, lower levels of FA appear to be driven by either higher levels of RD or lower levels of $A D$, and when $R D$ and $A D$ are both higher, no effect is observed in FA. Further research is required in order to more deeply understand this relationship in vivo. Sample size: chronic musculoskeletal pain patients $n=46$; healthy controls $n=33$. disorders ${ }^{66}$ ranging from schizophrenia, ${ }^{39}$ generalized anxiety, $^{55}$ and depression ${ }^{74}$ to temporal lobe epilepsy memory loss ${ }^{20}$ and Alzheimer's disease. ${ }^{80}$ Although the current study did not find statistically significant differences in uncinate $F A, R D$, or $A D$ between chronic musculoskeletal pain patients and healthy volunteers, the role that it clearly plays in processing emotion and memory makes it unsurprising that left uncinate FA was found to be correlated with both TOPS total pain experience and MPQ typical pain rating.

The posterior limb of the internal capsule conveys both ascending somatosensory and descending motor information. 1,11,23 The cluster of elevated RD reported above in this tract encompassed both sets of fibers. This increase may be attributable to abnormal processing of somatosensory input or pain-related behaviors such as decreased physical activity. Some evidence suggests that this pathway also contains descending frontal corticopontine fibers that play a role in pain inhibition. ${ }^{1,23}$

The superior longitudinal fasciculus and the cerebral peduncles (which also contain the descending corticospinal tracts) do not have an obvious role in the processing of chronic pain perception. However, both of these pathways are known to convey motor-related information, and changes in tract integrity in these pathways may reflect pain-related behavioral changes such as decreased physical activity. ${ }^{41,76}$ The cerebral peduncle was not selected a priori as a region of interest, but 
analyzed post hoc after a highly significant cluster of increased RD was observed there using TBSS. It is possible that like the posterior limbs of the internal capsule, the cerebral peduncles contain descending pain-inhibiting fibers, the properties of which may be affected in chronic pain.

Perhaps the most intriguing and novel findings reported here are the relationships between clinical measures and RD and AD. Based on theories of RD's role in neuroplasticity, we expected to find that RD was the most highly correlated measure of diffusion, yet no significant RD correlations were found. Perhaps even more interestingly, the-most correlations between metrics of diffusion and clinical measures involved $A D$. As it is not yet understood what factors influence $A D$ within a tract, the implications of these findings are unknown. Additional research is needed in order to determine how abnormalities in WM integrity contribute to and/ or result from chronic pain.

There are a few limitations that must be considered in interpreting our results. First, sample size is relatively small. Also, the demographic composition is skewed because the sample is predominantly female and ages are not perfectly balanced between groups. A broad sample of musculoskeletal pain diagnoses might be

\section{References}

1. Adams JE, Hosobuchi Y, Fields HL: Stimulation of internal capsule for relief of chronic pain. J Neurosurg 41:740-744, 1974

2. Alexander AL, Lee JE, Lazar M, Field AS: Diffusion tensor imaging of the brain. Neurotherapeutics 4:316-329, 2007

3. Anderson KO, Dowds BN, Pelletz RE, Thomas Edwards W, Peeters-Asdourian C: Development and initial validation of a scale to measure self-efficacy beliefs in patients with chronic pain. Pain 63:77-83, 1995

4. Anderson MJ, Robinson J: Permutation tests for linear models. Aust N Z J Stat 43:75-88, 2001

5. Andersson JL, Jenkinson M, Smith S: Non-linear Registration, aka Spatial Normalisation FMRIB Technical Report TR07JA2. FMRIB Analysis Group of the University of Oxford, Q6] 2007

6. Andersson JL, Jenkinson $\mathrm{M}$, Smith $\mathrm{S}$, Andersson J: Nonlinear Optimisation. FMRIB Technical Report TR07JA1. Oxford (UK), FMRIB Centre, 2007

7. Apkarian AV: Chronic back pain is associated with decreased prefrontal and thalamic gray matter density. J Neurosci 24:10410-10415, 2004

8. Baliki MN, Geha PY, Apkarian AV, Chialvo DR: Beyond feeling: Chronic pain hurts the brain, disrupting the defaultmode network dynamics. J Neurosci 28:1398-1403, 2008

9. Baliki MN, Schnitzer TJ, Bauer WR, Apkarian AV: Brain morphological signatures for chronic pain. PLoS One 6: e26010, 2011

10. Beck AT, Ward CH, Mendelson M, Mock J, Erbaugh J: An inventory for measuring depression. Arch Gen Psychiatry 4: 561,1961 considered as a strength, as it increases the generalizability of findings, but it might also prevent identification of phenomena specific to individual chronic pain diagnoses. Finally, additional confounds exist in the nature of our patient sample: Despite the fact that our patients were medically stable, even treated diabetes and hypertension may have influenced the properties of WM. It is also possible that stronger effects might have been observed if exclusion criteria did not render some patients with severe symptoms ineligible for analysis in this study (eg, for comorbid diagnoses of depression and anxiety disorders).

\section{Acknowledgments}

The authors would like the thank Jay Gonyea and Scott Hipko of the University of Vermont MRI Center for Biomedical Imaging for their assistance with scheduling and running scans, Dr. Shelly Naud for statistical advice, and the following individuals for their help acquiring, entering, and reconciling data: James Bishop, Nina La Rosa, Elizabeth McCallion, Hayley Perelman, Samuel Kellner, Emily Eck, Jenna Macarewicz, Alan French, and Dr. Asif Kalim.

11. Buckalew N, Haut MW, Aizenstein $H$, Morrow $L$, Perera S, Kuwabara H, Weiner DK: Differences in brain structure and function in older adults with self-reported disabling and nondisabling chronic low back pain. Pain Med 11:1183-1197, 2010

12. Buckalew N, Haut MW, Aizenstein $H$, Rosano $C$, Edelman KD, Perera S, Marrow L, Tadic S, Venkatraman V, Weiner D: White matter hyperintensity burden and disability in older adults: Is chronic pain a contributor? PM R 5:471-480, 2013

13. Buckalew N, Haut MW, Morrow L, Weiner D: Chronic pain is associated with brain volume loss in older adults: Preliminary evidence. Pain Med 9:240-248, 2008

14. Bullmore ET, Suckling J, Overmeyer $S$, Rabe-Hesketh $S$, Taylor E, Brammer MJ: Global, voxel, and cluster tests, by theory and permutation, for a difference between two groups of structural MR images of the brain. IEEE Trans Med Imaging 18:32-42, 1999

15. Burgmer $M$, Gaubitz $M$, Konrad C, Wrenger M, Hilgart S, Heuft G, Pfleiderer B: Decreased gray matter volumes in the cingulo-frontal cortex and the amygdala in patients with fibromyalgia. Psychosom Med 71:566-573, 2009

16. Burgmer M, Pogatzki-Zahn E, Gaubitz M, Wessoleck $E_{\text {, }}$ Heuft G, Pfleiderer B: Altered brain activity during pain processing in fibromyalgia. Neurolmage 44:502-508, 2009

17. Caminiti R, Carducci F, Piervincenzi C, Battaglia-Mayer A, Confalone G, Visco-Comandini F, Pantano P, Innocenti GM: Diameter, length, speed, and conduction delay of callosal axons in macaque monkeys and humans: Comparing data from histology and magnetic resonance imaging diffusion tractography. J Neurosci 33:14501-14511, 2013

18. Chen JY-W, Blankstein U, Diamant NE, Davis KD: White matter abnormalities in irritable bowel syndrome and relation to individual factors. Brain Res 1392:121-131, 2011 
19. Cook DB, Lange G, Ciccone DS, Liu WC, Steffener J, Natelson BH: Functional imaging of pain in patients with primary fibromyalgia. J Rheumatol 31:364, 2004

20. Diehl B, Busch RM, Duncan JS, Piao Z, Tkach J, Lüders HO: Abnormalities in diffusion tensor imaging of the uncinate fasciculus relate to reduced memory in temporal lobe epilepsy. Epilepsia 49:1409-1418, 2008

21. Ellingson BM, Mayer E, Harris RJ, Ashe-McNally C, Naliboff BD, Labus JS, Tillisch K: Diffusion tensor imaging (DTI) detects microstructural reorganization in the brain associated with chronic irritable bowel syndrome (IBS). Pain 154:1528-1541, 2013

22. Elliott AM, Smith BH, Penny KI, Cairns Smith W, Alastair Chambers W: The epidemiology of chronic pain in the community. Lancet 354:1248-1252, 1999

23. Fields $\mathrm{H}$, Adams J: Pain after cortical injury relieved by electrical stimulation of the internal capsule. Brain 97: 169-178, 1974

24. Folstein MF, Folstein SE, McHugh PR: "Mini-Mental State": A practical method for grading the cognitive state of patients for the clinician. J Psychiatr Res 12:189-198, 1975

25. Frøkjær JB, Olesen SS, Gram $M$, Yavarian $Y$, Bouwense SA, Wilder-Smith $\mathrm{OH}$, Drewes AM: Altered brain microstructure assessed by diffusion tensor imaging in patients with chronic pancreatitis. Gut 60:1554-1562, 2011

26. Geha PY, Baliki MN, Harden RN, Bauer WR, Parrish TB, Apkarian AV: The brain in chronic CRPS pain: Abnormal gray-white matter interactions in emotional and autonomic regions. Neuron 60:570-581, 2008

27. Gwilym SE, Filippini N, Douaud G, Carr AJ, Tracey I: Thalamic atrophy associated with painful osteoarthritis of the hip is reversible after arthroplasty: A longitudinal voxel-based morphometric study. Arthritis Rheum 62: 2930-2940, 2010

28. Han SD, Buchman AS, Arfanakis K, Fleischman DA, Bennett DA: Functional connectivity networks associated with chronic musculoskeletal pain in old age. Int J Geriatr Psychiatry 28:858-867, 2013

29. Harris PA, Taylor R, Thielke R, Payne J, Gonzalez N, Conde JG: Research electronic data capture (REDCap)-A metadata-driven methodology and workflow process for providing translational research informatics support. J Biomed Inform 42:377-381, 2009

30. Hasan KM, Iftikhar A, Kamali A, Kramer LA, Ashtari M, Cirino PT, Papanicolaou AC, Fletcher JM, Ewing-Cobbs L: Development and aging of the healthy human brain uncinate fasciculus across the lifespan using diffusion tensor tractography. Brain Res 1276:67-76, 2009

31. Hayasaka S, Nichols TE: Validating cluster size inference: Random field and permutation methods. Neurolmage 20: 2343-2356, 2003

32. Hua K, Zhang J, Wakana S, Jiang H, Li X, Reich DS, Calabresi PA, Pekar JJ, van Zijl P, Mori S: Tract probability maps in stereotaxic spaces: Analyses of white matter anatomy and tract-specific quantification. Neurolmage 39: 336-347, 2008

33. Hutchison WD, Davis K, Lozano A, Tasker R, Dostrovsky J: Pain-related neurons in the human cingulate cortex. Nat Neurosci 2:403-405, 1999
34. Jastak S, Wilkinson GS: The Wide Range Achievement Test-Revised: Administration manual. Washington, DC, Jastak Assessment Systems, 1984

35. Jenkinson M, Beckmann CF, Behrens TE, Woolrich MW, Smith SM: FSL. Neurolmage 62:782-790, 2012

36. Jones DK, Griffin LD, Alexander DC, Catani $M$, Horsfield MA, Howard R, Williams SCR: Spatial normalization and averaging of diffusion tensor MRI data sets. NeuroImage 17:592-617, 2002

37. Keihaninejad S, Ryan NS, Malone IB, Modat M, Cash D, Ridgway GR, Zhang H, Fox NC, Ourselin S: The importance of group-wise registration in Tract Based Spatial Statistics study of neurodegeneration: A simulation study in Alzheimer's disease. PLoS One 7:e45996, 2012

38. Kim J, Loggia ML, Edwards R, Wasan AD, Gollub RL, Napadow V: Sustained deep-tissue pain alters functional brain connectivity. Pain, 2013

39. Kubicki M, Westin C-F, Maier SE, Frumin M, Nestor PG, Salisbury DF, Kikinis $R$, Jolesz FA, McCarley RW, Shenton ME: Uncinate fasciculus findings in schizophrenia: A magnetic resonance diffusion tensor imaging study. Am J Psychiatry 159:813, 2002

40. Kuchinad A, Schweinhardt P, Seminowicz DA, Wood PB Chizh BA, Bushnell MC: Accelerated brain gray matter loss in fibromyalgia patients: Premature aging of the brain? J Neurosci 27:4004-4007, 2007

41. Leeuw M, Goossens ME, Linton SJ, Crombez G, Boersma K, Vlaeyen JW: The fear-avoidance model of musculoskeletal pain: Current state of scientific evidence. J Behav Med 30:77-94, 2007

42. Li XL, Fang YN, Gao QC, Lin EJ, Hu SH, Ren L, Ding $M H_{\text {, }}$ Luo BN: A diffusion tensor magnetic resonance imaging study of corpus callosum from adult patients with migraine complicated with depressive/anxious disorder. Headache 51: 237-245, 2011

43. Loggia ML, Kim J, Gollub RL, Vangel MG, Kirsch I, Kong J, Wasan AD, Napadow V: Default mode network connectivity encodes clinical pain: An arterial spin labeling study. Pain $154: 24-33,2013$

44. Lutz J, Jäger $L$, de Quervain $D$, Krauseneck $T$, Padberg $F$, Wichnalek M, Beyer A, Stahl R, Zirngibl B, Morhard D, Reiser M, Schelling G: White and gray matter abnormalities in the brain of patients with fibromyalgia: A diffusiontensor and volumetric imaging study. Arthritis Rheum 58: 3960-3969, 2008

45. Mansour AR, Baliki MN, Huang L, Torbey $S$, Herrmann KM, Schnitzer TJ, Apkarian AV: Brain white matter structural properties predict transition to chronic pain. Pain 154:2160-2168, 2013

46. Melzack R: The McGill Pain Questionnaire: Major properties and scoring methods. Pain 1:277-299, 1975

47. Melzack R: The short-form McGill Pain Questionnaire. Pain 30:191-197, 1987

48. Melzack R: The McGill Pain Questionnaire: From description to measurement. Anesthesiology 103:199-202, 2005

49. Moayedi M, Weissman-Fogel I, Salomons TV, Crawley AP, Goldberg MB, Freeman BV, Tenenbaum HC, Davis KD: Abnormal gray matter aging in chronic pain patients. Brain Res 1456:82-93, 2012 
50. Moayedi M, Weissman-Fogel I, Salomons TV, Crawley AP, Goldberg MB, Freeman BV, Tenenbaum HC, Davis KD: White matter brain and trigeminal nerve abnormalities in temporomandibular disorder. Pain 153:1467-1477, 2012

51. Mori S, Oishi $K$, Jiang $H$, Jiang $L$, Li X, Akhter $K$, Hua $K$, Faria AV, Mahmood A, Woods R: Stereotaxic white matter atlas based on diffusion tensor imaging in an ICBM template. Neurolmage 40:570-582, 2008

52. Mori S, Wakana S, Van Zijl PC, Nagae-Poetscher L: MRI atlas of human white matter. Philadelphia, Elsevier, 2005

53. Naylor MR, Helzer JE, Naud S, Keefe FJ: Automated telephone as an adjunct for the treatment of chronic pain: A pilot study. J Pain 3:429-438, 2002

54. Naylor MR, Keefe FJ, Brigidi B, Naud S, Helzer JE: Therapeutic interactive voice response for chronic pain reduction and relapse prevention. Pain 134:335-345, 2008

55. Phan KL, Orlichenko A, Boyd E, Angstadt M, Coccaro EF, Liberzon I, Arfanakis K: Preliminary evidence of white matter abnormality in the uncinate fasciculus in generalized social anxiety disorder. Biol Psychiatry 66:691-694, 2009

56. Rainville P, Duncan GH, Price DD, Carrier B, Bushnell MC: Pain affect encoded in human anterior cingulate but not somatosensory cortex. Science 277:968-971, 1997

57. Rodriguez-Raecke R, Niemeier A, Ihle K, Ruether W, May A: Brain gray matter decrease in chronic pain is the consequence and not the cause of pain. J Neurosci 29: 13746-13750, 2009

58. Rogers WH, Wittink HM, Ashburn MA, Cynn D, Carr DB: Using the "TOPS," an outcomes instrument for multidisciplinary outpatient pain treatment. Pain Med 1:55-67, 2000

59. Rogers WH, Wittink H, Wagner A, Cynn D, Carr DB: Assessing individual outcomes during outpatient multidisciplinary chronic pain treatment by means of an augmented SF-36. Pain Med 1:44-54, 2000

60. Schmidt-Wilcke $T$, Leinisch $E$, Gänßbauer $S$, Draganski B, Bogdahn U, Altmeppen J, May A: Affective components and intensity of pain correlate with structural differences in gray matter in chronic back pain patients. Pain 125:89-97, 2006

61. Schmidt-Wilcke T, Leinisch E, Straube A, Kämpfe N, Draganski B, Diener HC, Bogdahn U, May A: Gray matter decrease in patients with chronic tension type headache. Neurology 65:1483-1486, 2005

62. Schmidt-Wilcke $T$, Luerding $R$, Weigand $T$, Jürgens $T$, Schuierer G, Leinisch E, Bogdahn U: Striatal grey matter increase in patients suffering from fibromyalgia-A voxelbased morphometry study. Pain 132:S109-S116, 2007

63. Schreckenberger $M$, Siessmeier $T$, Viertmann $A$, Landvogt C, Buchholz HG, Rolke R, Treede RD, Bartenstein $\mathrm{P}$, Birklein $\mathrm{F}$ : The unpleasantness of tonic pain is encoded by the insular cortex. Neurology 64:1175-1183, 2005

64. Seminowicz DA, Shpaner $M$, Keaser $M L$, Krauthamer GM, Mantegna J, Dumas JA, Newhouse PA, Filippi C, Keefe FJ, Naylor MR: Cognitive-behavioral therapy increases prefrontal cortex gray matter in patients with chronic pain. J Pain 14:1573-1584, 2013

65. Seminowicz DA, Wideman TH, Naso L, HatamiKhoroushahi $Z$, Fallatah $S$, Ware MA, Jarzem $P$, Bushnell MC, Shir Y, Ouellet JA, Stone LS: Effective treatment of chronic low back pain in humans reverses abnormal brain anatomy and function. J Neurosci 31:7540-7550, 2011

66. Sexton CE, Mackay CE, Ebmeier KP: A systematic review of diffusion tensor imaging studies in affective disorders. Biol Psychiatry 66:814-823, 2009

67. Shackman AJ, Salomons TV, Slagter HA, Fox AS, Winter JJ, Davidson RJ: The integration of negative affect, pain and cognitive control in the cingulate cortex. Nat Rev Neurosci 12:154-167, 2011

68. Smith SM: Fast robust automated brain extraction. Hum Brain Mapp 17:143-155, 2002

69. Smith $S M$, Jenkinson $M$, Johansen-Berg $H$, Rueckert $D_{\text {, }}$ Nichols TE, Mackay CE, Watkins KE, Ciccarelli O, Cader MZ, Matthews PM: Tract-based spatial statistics: Voxelwise analysis of multi-subject diffusion data. Neurolmage 31: 1487-1505, 2006

70. Smith $S M$, Jenkinson $M$, Woolrich MW, Beckmann $C F$ Behrens TE, Johansen-Berg $H$, Bannister PR, De Luca $M$, Drobnjak I, Flitney DE: Advances in functional and structural MR image analysis and implementation as FSL. Neurolmage 23:S208-S219, 2004

71. Sullivan MJ, Bishop SR, Pivik J: The Pain Catastrophizing Scale: Development and validation. Psychol Assess 7:524, 1995

72. Sundgren $P C$, Petrou $M$, Harris RE, Fan $X$, Foerster $B$, Mehrotra N, Sen A, Clauw DJ, Welsh RC: Diffusion-weighted and diffusion tensor imaging in fibromyalgia patients: $A$ prospective study of whole brain diffusivity, apparent diffusion coefficient, and fraction anisotropy in different regions of the brain and correlation with symptom severity. Acad Radiol 14:839, 2007

73. Szabó N, Kincses ZT, Párdutz Á, Tajti J, Szok D, Tuka B, Király A, Babos M, Vörös E, Bomboi G: White matter microstructural alterations in migraine: A diffusion-weighted MRI study. Pain 153:651-656, 2012

74. Taylor WD, MacFall JR, Gerig G, Krishnan RR: Structural integrity of the uncinate fasciculus in geriatric depression: Relationship with age of onset. Neuropsychiatr Dis Treat 3: 669, 2007

75. Treede R-D, Apkarian AV, Bromm B, Greenspan JD, Lenz FA: Cortical representation of pain: Functional characterization of nociceptive areas near the lateral sulcus. Pain $87: 113-119,2000$

76. Vlaeyen J, Crombez G: Fear of movement/(re)injury, avoidance and pain disability in chronic low back pain patients. Man Ther 4:187-195, 1999

77. Vogt BA: Pain and emotion interactions in subregions of the cingulate gyrus. Nat Rev Neurosci 6:533-544, 2005

78. Vogt BA, Sikes RW, Vogt LJ: Anterior cingulate cortex and the medial pain system, in Vogt BA, Gabriel M (eds): Neurobiology of Cingulate Cortex and Limbic Thalamus. Boston, Birkhäuser, 1993, pp 313-344

79. Woolrich MW, Jbabdi S, Patenaude B, Chappell $M$, Makni S, Behrens T, Beckmann C, Jenkinson M, Smith SM: Bayesian analysis of neuroimaging data in FSL. Neurolmage 45:S173-S186, 2009

80. Yasmin H, Nakata $Y$, Aoki S, Abe O, Sato N, Nemoto K, Arima K, Furuta N, Uno M, Hirai S: Diffusion abnormalities of the uncinate fasciculus in Alzheimer's disease: Diffusion tensor tract-specific analysis using a new method to measure the core of the tract. Neuroradiology 50:293-299, 2008 